\title{
Bilateral subtrochanteric femoral fractures in a previous alendronate-medicated patient: a case report
}

This article was published in the following Dove Press journal:

Clinical Audit

5 August 2014

Number of times this article has been viewed

\author{
Jacob J Triplet ${ }^{1}$ \\ Timothy Hawkes ${ }^{2}$ \\ Sein Lwin ${ }^{2}$ \\ 'Nova Southeastern University, \\ College of Osteopathic Medicine, Fort \\ Lauderdale, FL, USA; ${ }^{2}$ Broward Health \\ Medical Center, Orthopedic Surgery \\ Residency Program, Fort Lauderdale, \\ FL, USA
}

\begin{abstract}
Bisphosphonate therapy is commonly employed in the management of osteoporosis. Several drug-associated adverse events are well established but are often not considered in patients who have discontinued therapy. Here, we present the case of a patient who sustained bilateral atypical subtrochanteric fractures 1 year after discontinuation of long-term bisphosphonate therapy. We discuss the prodromal clinical presentations and the importance of radiographic imaging to investigate impending fractures. The treatment of these fractures included the implementation of a drug holiday and the use of intramedullary nail stabilization.
\end{abstract}

Keywords: bisphosphonate, femur, osteoporosis, prophylactic stabilization

\section{Introduction}

Bone density, and its associated fragility fractures, remains clinically challenging to treat in the elderly population. The National Osteoporosis Foundation's 2013 prevalence data reported an estimated 9 million US adults with osteoporosis, and another 48 million being osteopenic. ${ }^{1}$ Bisphosphonate therapy plays a fundamental role in the management of osteoporosis, with more than 190 million prescriptions for oral bisphosphonates dispensed worldwide each year. ${ }^{2}$ The effectiveness of this therapy is attributed to its ability to reduce bone resorption by way of osteoclast inhibition. Alendronate is a common first-line choice due to its proven effectiveness, tolerability, and low cost. ${ }^{3}$

Long-term bisphosphonate therapy is not without consequence. Known side effects include gastrointestinal upset, musculoskeletal pain, associated low-energy fractures of the proximal femur, and osteonecrosis of the jaw. ${ }^{3}$ Bisphosphonate-associated adverse effects are often disregarded in patients who have discontinued therapy. Here, we present the case of a patient who sustained bilateral atypical subtrochanteric femoral fractures 1 year after discontinuation of long-term bisphosphonate therapy. It is important to note that although atypical subtrochanteric femoral fractures are an associated characteristic of bisphosphonate therapy, they also occur in patients without a history of bisphosphonate therapy. ${ }^{4}$ The prodromal clinical presentation, the importance of radiographic imaging to investigate impending fractures, the implementation of a drug holiday, and the use of intramedullary nail stabilization for fracture prophylaxis, as recommended in the literature, are all discussed. Written informed consent was obtained from the patient for publication of this case report and the accompanying images.

\section{Case report}

A 71-year-old Caucasian female presented to the emergency room following a trip-andfall from standing height onto her right side. She was unable to ambulate, and dysfunction 
of her right lower extremity was noted. The patient's history consists of hypertension, type 2 diabetes mellitus, osteoporosis, dyslipidemia, and generalized anxiety disorder. Current medications included atorvastatin, hydrochlorothiazide, losartan, venlafaxine, metoprolol, and a multivitamin. She was previously taking alendronate for a period of 10 years following a diagnosis of osteoporosis. Prior to the initiation of bisphosphonate therapy, this patient had sustained a fracture to the right distal radius. She had discontinued the bisphosphonate therapy 1 year prior to the incident at the discretion of her primary care physician. Upon further questioning, the patient stated that she had pain in the right thigh months prior to the incident, in the absence of trauma. The patient denied any prior pain in the left thigh.

Radiographs demonstrated a varus angulated transverse subtrochanteric fracture with lateral cortical thickening of the

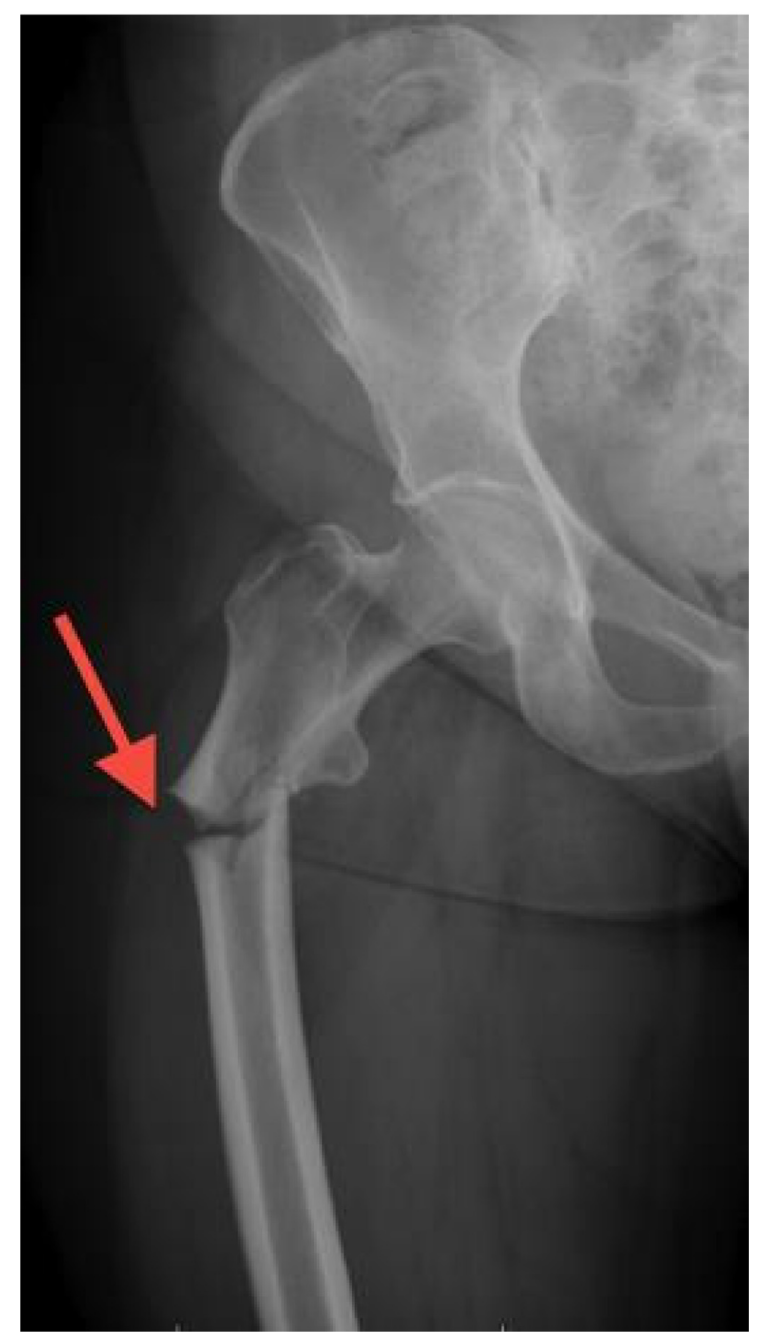

Figure I Anteroposterior radiograph of a varus angulated transverse subtrochanteric fracture with lateral cortical thickening of the right femur.

Note: The arrow demonstrates the fracture line. right femur (Figure 1). A radiograph taken of the opposite left femur revealed a small incomplete transverse subtrochanteric fracture with lateral cortical thickening (Figure 2). Orthopedic examination showed tenderness to palpation over the greater trochanter with swelling to the right thigh. Pain was present with all movements of the involved extremities. The patient's neurovascular examination was unremarkable.

After discussion of the risks and benefits of nonoperative and operative treatment options, the patient elected to undergo bilateral surgical stabilization. Surgical stabilization of the right subtrochanteric fracture was managed

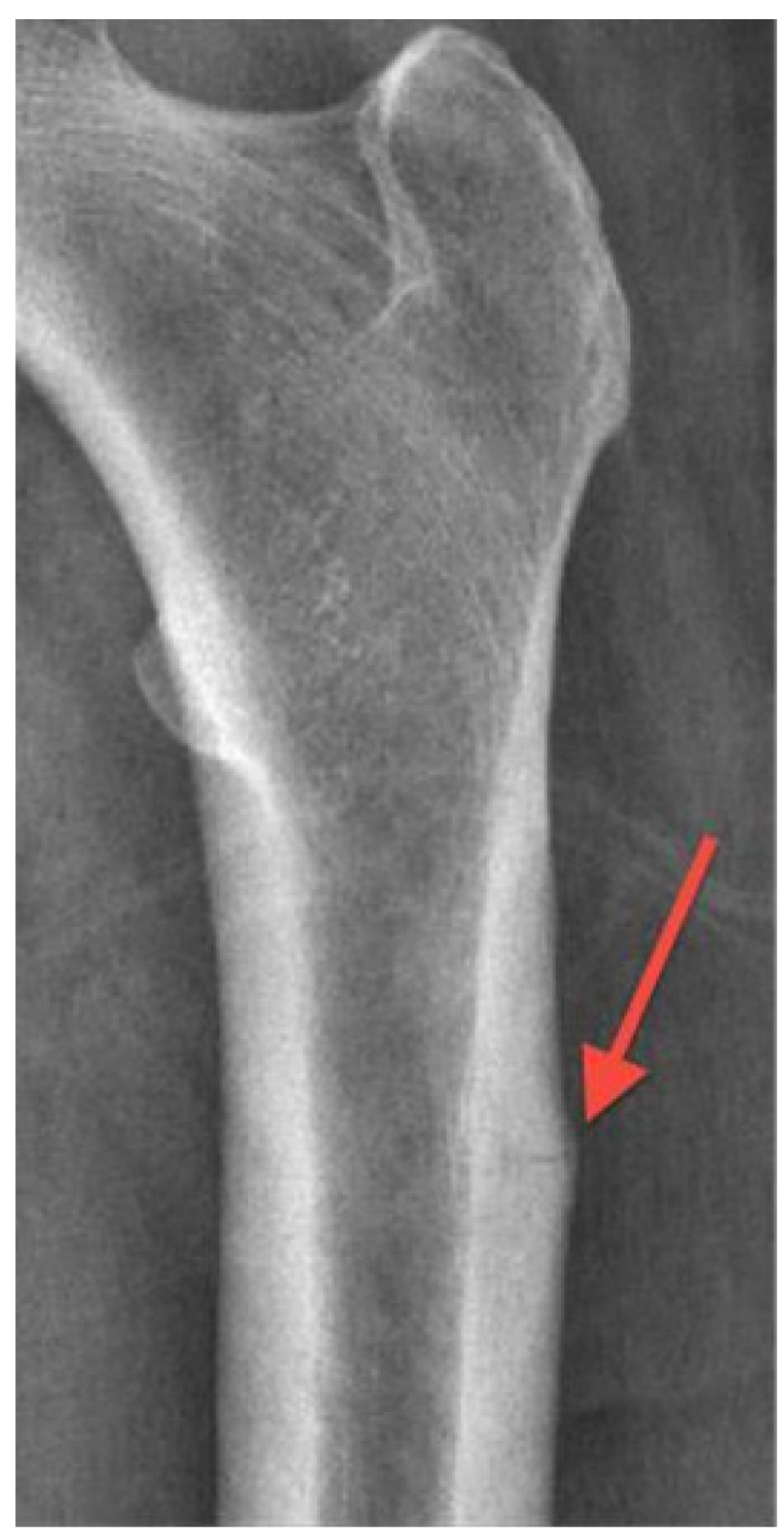

Figure 2 Magnification of a small, incomplete subtrochanteric fracture with lateral cortical thickening of the left femur.

Note: The arrow demonstrates the fracture line. 
with an antegrade intramedullary nail. The left femur was prophylactically managed with antegrade intramedullary orthopedic fixation. Postoperatively, the patient was allowed to partially weight-bear on the right lower extremity and fully weight-bear on the left lower extremity as tolerated. The patient was discharged home and remains under orthopedic care for follow-up evaluations.

\section{Discussion}

The American Society for Bone and Mineral Research has established diagnostic criteria for identification of atypical femoral fractures. ${ }^{5}$ Occurrences of these atypical femoral fractures have been reported in patients on bisphosphonate therapy. ${ }^{6}$ Additionally, this case demonstrates that this association may continue at least 1 year following discontinuation of previous long-term bisphosphonate therapy. Here, the associated subtrochanteric fracture pattern, bilateral involvement, prodromal symptoms, and characteristic cortical thickening are demonstrated in a patient following cessation of longterm therapy. ${ }^{7-9}$

Lifestyle recommendations such as weight-bearing exercise, sufficient calcium and vitamin D intake, fall prevention, and smoking cessation are fundamental in the prevention of osteoporosis. However, these practices are often not enough in an osteoporotic patient. ${ }^{3}$ Originally synthesized in the $1800 \mathrm{~s}$, bisphosphonates were not being investigated for their use in the treatment of osteoporosis until the 1970s and 1980s. ${ }^{3}$ Their effectiveness is attributed to their ability to reduce bone resorption via osteoclast inhibition, minimizing bone loss and improving bone density. ${ }^{10,11}$ The two phosphate groups bind calcium in bone, allowing for accumulation and attainment of high concentrations. This inhibition of osteoclasts decreases resorption of both cortical and trabecular bone. The differences in Young's modulus between the thickened cortical bone and the less dense trabecular bone may lead to a stress riser and subsequent stress fracture. ${ }^{12}$ The more reactive trabecular bone may exacerbate the differences in stiffness and the structural properties of the bone types, ${ }^{12}$ even after cessation of bisphosphonate treatment.

In a previous report of complete femoral fractures, Goh et $\mathrm{al}^{7}$ described the incidence of prolonged suppression of bone remolding with alendronate use. This phenomenon, coupled with the high stress forces on the proximal femur, provides an environment for stress fracture formation. These mechanical stresses result from a combination of body weight and deforming forces exerted by multiple muscles. Considerable bending forces are applied at the proximal femur due to the angle of the applied forces being perpendicular to the axis of the femoral neck.

Clinically, long-term bisphosphonate-medicated patients who sustain proximal femoral stress fractures often present with prodromal symptoms, such as thigh pain in the absence of trauma. ${ }^{12}$ These stress fractures characteristically have a transverse or oblique pattern. Long-term therapy may lead to severely suppressed bone turnover, increasing susceptibility to these nonspiral fractures. ${ }^{13}$ The transverse nature of these fractures may increase the healing time. Radiological features such as lateral cortical thickening may also be evident..$^{7-9,12}$ In a study of 15 complete simple or oblique fractures, Lenart et $\mathrm{al}^{9}$ demonstrated that patients with beaking and diffuse thickening of the cortex had longer alendronate use than those without this pattern $(7.3 \pm 1.8$ years and $2.8 \pm 1.3$ years, respectively); these findings have been supported. ${ }^{8}$ Due to this association, close follow-up visits and a high level of suspicion may prevent the development of a future complete fracture. ${ }^{3}$ Radiographs are recommended to detect early stress reactions. ${ }^{14}$

Patients receiving or having received long-term bisphosphonate therapy should be counselled to report the occurrence of new thigh or groin pain so that appropriate evaluation and management may be implemented prior to the potential occurrence of a complete fracture. Occurrence of an atypical femur fracture should raise concern regarding an impeding contralateral fracture, and appropriate imaging should be obtained.

Subtrochanteric femoral fractures may be effectively managed though a variety of treatment modalities, both surgical and nonsurgical. In patients who present with subtrochanteric femoral fractures, intramedullary nail stabilization has been shown to be an appropriate treatment. ${ }^{15}$ Incomplete bisphosphonate-related femoral fractures have also been successfully treated nonoperatively. However, a higher percentage of patients who are treated surgically show radiographic evidence of earlier healing. ${ }^{15,16}$ Prophylactic surgical intervention was shown to provide symptomatic relief and improve functional status. ${ }^{16}$

In a study of 43 incomplete bisphosphonate-associated femoral fractures, it was reported that $100 \%$ of patients treated surgically and $18 \%$ of patients treated nonoperatively were radiographically healed at a mean of 7.1 months and 11 months, respectively. ${ }^{16}$ In the case of bilateral involvement, prophylactic stabilization may be considered both for fracture prevention and allowance of earlier weightbearing mobilization. Patients who have sustained longterm bisphosphonate-associated femur fractures may also 
benefit from alternative medication. Gomberg et al ${ }^{17}$ have reported that teriparatide, an anabolic agent, in addition to supplemental calcium, vitamin D therapy, and alendronate discontinuation, may result in the healing of a long-term alendronate-associated fracture.

Studies have validated the effectiveness of bisphosphonates even after their discontinuation. ${ }^{18,19}$ Somford et $\mathrm{al}^{18}$ demonstrated that bone density mineralization was higher than pretreatment levels 5 years after discontinuation of the bisphosphonates. Black et $\mathrm{al}^{19}$ revealed only a slight increase in vertebral fractures after 5 years of discontinuation. Accordingly, this case suggests that the adverse sequelae associated with long-term bisphosphonate use may also persist following its discontinuation. In a retrospective review of 59 atypical femoral fractures, Schilcher et $\mathrm{al}^{4}$ demonstrated that after bisphosphonate withdrawal, a fracture risk reduction of $70 \%$ per year is evident.

Due to the occurrence of atypical femoral fractures associated with long-term bisphosphonate use and a rapid decline in fracture risk following therapy withdrawal, ${ }^{4}$ a more constrained usage, as well as the implementation of a drug holiday of 2-3 years after the initial treatment of 5 years, is warranted for low-to-moderate risk patients, as recommended in the literature..$^{3,4,20-22}$ However, the duration to optimize efficacy for fracture risk reduction with bisphosphonate therapy is ultimately based on the individual assessment of patient preferences, risks and benefits. ${ }^{21}$ Black et $\mathrm{al}^{22}$ have reported incidences in which continuation of therapy after $3-5$ years may be beneficial. For patients who have a low bone mass density at the femoral neck, a T-score below -2.5 and who are at high risk for vertebral fractures, continuation of therapy may be beneficial. ${ }^{22}$ Additionally, patients with an existing vertebral fracture and a T-score no higher than -2.0 may also benefit from continued bisphosphonate therapy.22

\section{Conclusion}

Clinicians must not disregard bisphosphonate-associated adverse sequelae in patients who have discontinued therapy, and should suspect potential impending fractures in these patients following unexplained upper thigh or groin pain. Radiographic imaging may be utilized to investigate impending fractures. A more constrained usage, as well as the implementation of a drug holiday of 2-3 years following the initial treatment of 5 years, is recommended in low-to-moderate fracture risk patients, ${ }^{3,4,20-22}$ as bone density mineralization has been shown to be higher than pretreatment levels 5 years after discontinuation of the bisphosphonates. ${ }^{18}$
Prophylactic surgical stabilization with an intramedullary nail may be considered for incomplete fractures, as it has been demonstrated in the literature to result in earlier healing, symptomatic relief, and improved functional status when compared with nonoperative alternatives. ${ }^{16}$

\section{Acknowledgment}

We would like to thank Dr Isa Fernandez for her guidance and support.

\section{Author contributions}

Jacob J. Triplet composed this manuscript and was involved in the data analysis and the drafting of the manuscript. Sein Lwin and Timothy Hawkes performed the surgeries described in this case report, were involved in the design and acquisition of the data, as well as the revision of the manuscript. All authors have reviewed and agree upon the submission of this manuscript.

\section{Disclosure}

The authors report no conflicts of interest in this work.

\section{References}

1. National Osteoporosis Foundation. NOF releases new data detailing the prevalence of osteoporosis. 2013. Available from: http://nof.org/files/ nof/public/content/file/2158/upload/875.pdf. Accessed July 8, 2014.

2. Otto S, Abu-Id MH, Fedele S, et al. Osteoporosis and bisphosphonatesrelated osteonecrosis of the jaw: not just a sporadic coincidence: a multi-centre study. J Craniomaxillofac Surg. 2011;39:272-277.

3. Lems WF, den Heijer M. Established and forthcoming drugs for the treatment of osteoporosis. Neth J Med. 2013;71:188-193.

4. Schilcher J, Michaëlsson K, Aspenberg P. Bisphosphonate use and atypical fractures of the femoral shaft. N Engl J Med. 2011;364(18): $1728-1737$.

5. Shane E, Burr D, Ebeling PR, et al. Atypical subtrochanteric and diaphyseal femoral fractures: report of a task force of the American Society of Bone and Mineral Research. J Bone Miner Res. 2010;25:2267-2294.

6. Selvan S, Mills T, McNally J. Audit of atypical hip fractures at the Royal Berkshire Hospital. Clinical Audit. 2013;5:91-93.

7. Goh SK, Yang KY, Koh JS, et al. Subtrochanteric insufficiency fractures in patients on alendronate therapy: a caution. J Bone Joint Surg Br. 2007;89:349-353.

8. Neviaser AS, Lane JM, Lenart BA, et al. Low-energy femoral shaft fractures associated with alendronate use. J Orthop Trauma. 2008;22: 346-350.

9. Lenart BA, Lorich DG, Lane JM. Atypical fractures of the femoral diaphysis in postmenopausal women taking alendronate. $N$ Engl J Med. 2008;358:1304-1306

10. Pols HA, Felsenberg D, Hanley DA, et al. Multinational, placebocontrolled, randomized trial of the effects of alendronate on bone density and fracture risk in postmenopausal women with low bone mass: results of the FOSIT study. Fosamax International Trial Study Group. Osteoporos Int. 1999;9:461-468.

11. Liberman UA, Weiss SR, Broll J, et al. Effect of oral alendronate on bone mineral density and the incidence of fractures in postmenopausal osteoporosis. The Alendronate Phase III Osteoporosis Treatment Study Group. N Engl J Med. 1995;333:1437-1443. 
12. Kumar G, Dunlop C. Effects of bilateral distal femoral stress in a patient on long-term pamidronate. Am J Orthop. 2013;7:326-328.

13. Cheung RK, Leung KK, Lee KC, et al. Sequential non-traumatic femoral shaft fractures in a patient on long-term alendronate. Hong Kong Med J. 2007;13:485-489.

14. Kwek EB, Goh SK, Koh JS, et al. An emerging pattern of subtrochanteric stress fractures: a long-term complication of alendronate therapy? Injury. 2008;39:224-231.

15. Das De S, Setiobudi T, Shen L, et al. A rational approach to management of alendronate-related subtrochanteric fractures. J Bone Joint Surg Br. 2010;92:679-686.

16. Egol KA, Park JH, Prensky C, et al. Surgical treatment improves clinical and functional outcomes for patients who sustain incomplete bisphosphonate-related femur fractures. J Orthop Trauma. 2013;27: 331-335.

17. Gomberg SJ, Wustrack RL, Napoli N, Arnaud CD, Black DM. Teriparatide, vitamin $\mathrm{D}$, and calcium healed bilateral subtrochanteric stress fractures in a postmenopausal woman with a 13-year history of continuous alendronate therapy. J Clin Endocrinol Metab. 2011;96(6): $1627-1632$.
18. Somford MP, Geurts GF, den Teuling JW, et al. Long-term alendronate use not without consequences? Int J Rheumatol. 2009;2009:253432.

19. Black DM, Schwartz AV, Ensrud KE, et al. Effects of continuing or stopping alendronate after 5 years of treatment: the Fracture Intervention Trial Long-term Extension (FLEX): a randomized trial. JAMA. 2006;296:2927-2938.

20. Ott SM. What is the optimal duration of bisphosphonate therapy? Cleve Clin J Med. 2011;78:619-630.

21. Whitaker M, Guo J, Kehoe T, Benson G. Bisphosphonates for osteoporosis: where do we go from here? N Engl J Med. 2012;366(22): 2048-2051.

22. Black DM, Bauer DC, Schwartz AV, Cummings SR, Rosen CJ. Continuing bisphosphonate treatment for osteoporosis: for whom and for how long? N Engl J Med. 2012;366(22):2051-2053.
Clinical Audit

\section{Publish your work in this journal}

Clinical Audit is an international, peer-reviewed, open access journal focusing on the processes and outcomes of clinical audit in any area of healthcare. All aspects of patient care are addressed within the journal and practitioners from all disciplines are invited to submit their work. Areas covered include: Publication of audits; How an audit has changed practice;

\section{Dovepress}

Practical tips on how to do audits and to avoid pitfalls; How audits have changed patient care; Calls and justifications for new audits. The manuscript management system is completely online and includes a very quick and fair peer-review system, which is all easy to use. Visit http://www.dovepress. com/testimonials.php to read real quotes from published authors.

Submit your manuscript here: http://www.dovepress.com/clinical-audit-journal 